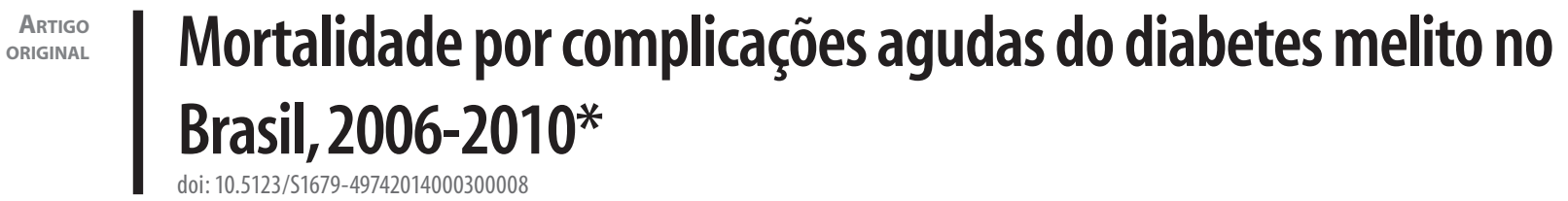

\author{
Mortality from acute complications of diabetes mellitus in Brazil, 2006-2010
}

\author{
André Klafke \\ Programa de Pós-Graduação em Epidemiologia, Universidade Federal do Rio Grande do Sul e Serviço de Saúde Comunitária, \\ Grupo Hospitalar Conceição, Porto Alegre-RS, Brasil
}

Bruce Bartholow Duncan

Programa de Pós-Graduação em Epidemiologia, Universidade Federal do Rio Grande do Sul, Porto Alegre-RS, Brasil

Roger dos Santos Rosa

Programa de Pós-Graduação em Epidemiologia, Universidade Federal do Rio Grande do Sul, Porto Alegre-RS, Brasil

\title{
Lenildo de Moura
}

Programa de Pós-Graduação em Epidemiologia, Universidade Federal do Rio Grande do Sul, Porto Alegre-RS e Unidade Técnica de Determinantes Sociais e Riscos para a Saúde, Doenças Crônicas Não Transmissíveis e Saúde Mental, Organização PanAmericana da Saúde, Brasília-DF, Brasil

Deborah Carvalho Malta

Secretaria de Vigilância em Saúde, Ministério da Saúde, Brasília-DF, Brasil

Maria Inês Schmidt

Programa de Pós-Graduação em Epidemiologia, Universidade Federal do Rio Grande do Sul, Porto Alegre-RS, Brasil

\section{Resumo}

Objetivo: descrever a mortalidade por complicações agudas do diabetes melito no Brasil segundo faixa etária, sexo, regiões e unidades federativas. Métodos: estudo descritivo com dados de óbitos registrados no Sistema de Informações sobre Mortalidade (SIM), corrigidos para sub-registro e causas mal definidas, para 2010 ou o período 2006-2010; as taxas foram padronizadas por idade e sexo. Resultados: a mortalidade por complicações agudas no Brasil foi de 2,45/100 mil habitantes e de 0,29/100 mil hab. entre menores de 40 anos de idade, correspondendo a 6,8\% e 22,9\% dos óbitos pelo diabetes como causa básica, respectivamente; a taxa de mortalidade foi maior nas regiões Norte (4,33/100 mil) e Nordeste (3,46/100 mil), aumentando com a idade. Conclusões: a taxa de mortalidade por complicações agudas do diabetes foi elevada, especialmente no Norte e Nordeste, considerando-se sua potencial evitabilidade; este indicador mostrou-se importante para avaliação de ações preventivas e de iniquidades regionais em saúde.

Palavras-chave: Diabetes Mellitus; Complicações do Diabetes; Coma Diabético; Cetoacidose Diabética; Epidemiologia Descritiva.

\begin{abstract}
Objective: to describe mortality from acute complications of diabetes in Brazil according to geographical region, age and sex. Methods: deaths registered on the Brazilian Mortality System due to these complications (ICD-10 E1014.0 or 1) in 2006-2010 were corrected for under-reporting and ill-defined causes. Mortality rates were standardized by age and sex. Results: annual mortality in Brazil was 2.45 deaths per 100,000 inhabitants, and 0.29 deaths/100,000 inhabitants among those $<40$ years old, accounting for $6.8 \%$ and $22.9 \%$ of deaths registering diabetes as the underlying cause, respectively. Mortality was higher in the North and Northeast regions and increased with age. Conclusion: given its potential avoidability, mortality due to acute complications of diabetes was high, especially in the Northeast and the North regions. As such, it is an important indicator for evaluating preventive actions and regional health inequalities.

Key words: Diabetes Mellitus; Diabetes Complications; Diabetic Coma; Diabetic Ketoacidosis; Descriptive Epidemiology.
\end{abstract}

\footnotetext{
* Este manuscrito originou-se de tese desenvolvida por André Klafke para a conclusão do curso de Doutorado em Epidemiologia pelo Programa de Pós-Graduação em Epidemiologia da Universidade Federal do Rio Grande do Sul (UFRGS), defendida em 2013.O estudo foi financiado com recursos previstos pelo Termo de Cooperação n $80 / 2009$, firmado entre a UFRGS e o Ministério da Saúde.
}

Endereco para correspondência:

André Klafke - Universidade Federal do Rio Grande do Sul, Faculdade de Medicina, Programa de Pós-Graduação em

Epidemiologia, Rua Ramiro Barcelos, n₹2600, sala 419, bairro Rio Branco, Porto Alegre-RS, Brasil. CEP: 90035-003

E-mail:andreklafke@hotmail.com 


\section{Introdução}

Nas últimas décadas, a epidemia mundial de diabetes melito tem representado um desafio para os sistemas de saúde. Estimativas recentes indicaram que 11,9 milhões de pessoas entre 20 e 79 anos de idade tinham diabetes no Brasil, ${ }^{1}$ tornando o país o quarto em número de casos de diabetes no mundo.

As complicações agudas do diabetes melito incluem a cetoacidose diabética, o estado hiperglicêmico hiperosmolar e a hipoglicemia. Os episódios de estado hiperglicêmico hiperosmolar e os de cetoacidose apresentam elevada letalidade, cerca de 15 e 5\% respectivamente. ${ }^{2}$ Esses episódios são acompanhados de importante sintomatologia, como perda de peso, coma e desidratação, e seu manejo gera altos custos para os pacientes e para a sociedade. ${ }^{3}$

\section{As complicações agudas do diabetes melito incluem a cetoacidose diabética, o estado hiperglicêmico hiperosmolar e a hipoglicemia.}

A grande maioria dos episódios de complicações agudas poderia ser evitada, na medida em que decorre de dificuldades no acesso aos serviços de saúde, incluindo o pronto atendimento a essas complicações, inadequada distribuição de insulina, omissão de dose de insulina e infecções agudas. ${ }^{4-6}$ Diante dessa realidade nacional e do reconhecimento internacional da importância crescente do diabetes como problema de Saúde Pública, ${ }^{7}$ além da ausência de dados populacionais publicados sobre 0 assunto no Brasil, o presente estudo tem por objetivo descrever a mortalidade por complicações agudas do diabetes melito no país segundo faixa etária, sexo, regiões e unidades da federação (UF) em 2010 - ou no período 2006-2010.

\section{Métodos}

Foi realizado um estudo descritivo sobre mortalidade a partir dos óbitos notificados ao Sistema de Informações sobre Mortalidade (SIM) e de dados demográficos disponibilizados pelo Instituto Brasileiro de Geografia e Estatística (IBGE), referentes ao ano 2010 ou ao período 2006-2010, quando indicado.
Foram considerados óbitos devidos ao diabetes todos os óbitos ocorridos no território brasileiro, declarados e registrados no SIM, que contivessem, no item 'causa do óbito', o código de um dos três caracteres previstos na $10^{a}$ Revisão da Classificação Estatística Internacional de Doenças e Problemas Relacionados à Saúde (CID-10): E10 (diabetes mellitus insulinodependente); E11 (diabetes mellitus não insulinodependente); E12 (diabetes mellitus relacionado com a desnutrição); E13 (outros tipos especificados de diabetes mellitus); ou E14 (diabetes mellitus não especificado). Foram considerados óbitos declarados por complicações agudas do diabetes todos os óbitos que contivessem, além de um dos critérios acima, o código de quarto caractere $\mathbf{0}$ [coma diabético com ou sem cetoacidose, coma diabético hiperosmolar, coma diabético hipoglicêmico ou coma hiperglicêmico sem outra especificação (SOE)] ou $\mathbf{1}$ (acidose diabética ou cetoacidose diabética sem menção de coma). Foram considerados óbitos precoces por complicações agudas do diabetes todos os óbitos por essas complicações ocorridos antes dos 40 anos de idade.

A população do Brasil e de suas grandes regiões foi considerada a população residente na respectiva localidade, conforme o Censo Demográfico 2010, realizado e disponibilizado pelo IBGE. Devido ao menor número de óbitos levantados nas análises por UF, e nas regiões Norte, Centro-Oeste e Sul na mortalidade precoce, foram utilizados os óbitos de 2006 a 2010 no cálculo das taxas de mortalidade por UF e de mortalidade precoce por região. A população de 2006 a 2009 foi estimada por interpolação linear entre as populações dos anos 2000 e 2010.

Os óbitos declarados por diabetes e suas complicações agudas foram corrigidos pela presença de óbitos declarados por causas mal definidas (capítulo XVIII da CID-10), pressupondo que sua distribuição seja equivalente à dos óbitos declarados por causas definidas não externas, conforme orientação da Organização Mundial da Saúde (OMS) ${ }^{8}$ Os dados foram então corrigidos para sub-registro, a partir das estimativas de subnotificação. ${ }^{9,10}$

As taxas de mortalidade para 2010 foram calculadas dividindo-se o total de óbitos pela população correspondente daquele ano. Para as UF e para a mortalidade precoce nas regiões, foi calculada a média das taxas de mortalidade anuais de 2006 a 2010. Para fins de comparabilidade internacional, as taxas de mortalidade foram padronizadas para idade e sexo via método direto, considerando-se como padrão a população mundial, conforme orientação da OMS. ${ }^{8,9}$ 
A razão de taxas entre os sexos foi calculada dividindose as taxas feminina/masculina, padronizadas por idade, para 0 agregado das faixas etárias. A significância estatística na comparação entre duas taxas foi avaliada mediante 0 intervalo de confiança de $95 \%$ ( IC $_{95 \%}$ ) da razão de taxas. ${ }^{11}$ Os dados foram analisados pelo programa Stata 10.0.

Os dados utilizados são de domínio público, disponibilizados pelo Departamento de Informática do SUS (Datasus) em meio eletrônico, sem identificação dos sujeitos. 0 projeto do estudo foi aprovado pelo Comitê de Ética em Pesquisa do Hospital de Clínicas de Porto Alegre sob o ${ }^{\circ}$ 100.056, e atende à Resolução do Conselho Nacional de Saúde (CNS) n 466, de 12 de dezembro de 2012.

\section{Resultados}

Em 2010, ocorreram 3.741 óbitos por complicações agudas do diabetes no Brasil (Tabela 1), correspondendo a $6,8 \%\left(\mathrm{IC}_{95 \%}: 6,6 ; 7,0 \%\right)$ dos 54.857 óbitos por diabetes como causa básica no país. Os óbitos se distribuíram de forma similar, entre 'coma' e 'cetoacidose sem menção de coma'; sua grande maioria $(79,9 \%)$ não apresentava, em seu registro, especificação do tipo de diabetes (75,7\% em menores de 40 anos e 79,7\% naqueles com 60 ou mais anos de idade). A taxa de mortalidade por complicações agudas do diabetes no país, após correções e padronizações, foi de 2,45 (IC ${ }_{95 \%}$ : 2,38; 2,52) óbitos por 100 mil habitantes (Tabela 2).

Considerando-se apenas os menores de 40 anos de idade, ocorreram 313 óbitos por complicações agudas do diabetes no Brasil no ano de 2010, correspondendo a $22,9 \%\left(\mathrm{IC}_{95 \%}: 20,7 ; 25,1 \%\right)$ dos 1.367 óbitos com menção de diabetes como causa básica. A taxa de mortalidade por essas complicações em menores de 40 anos foi de 0,29 (IC $\left._{95 \%}: 0,26 ; 0,32\right)$ óbitos por $100 \mathrm{mil}$ habitantes naquele ano, no país (Tabela 2 ).

A mortalidade por complicações agudas aumentou exponencialmente com a idade, partindo de quase zero, na faixa etária de menos de cinco anos, e chegando a 39,6 óbitos por 100 mil habitantes $\left(\mathrm{IC}_{95 \%}: 37,3 ; 41,8\right.$ ) na faixa etária dos 80 ou mais anos (Figura 1). A taxa de mortalidade atingiu o menor valor entre os cinco e os nove anos de idade, com $0,07\left(\mathrm{IC}_{95 \%}: 0,03 ; 0,11\right)$ óbitos por 100 mil habitantes. A mortalidade por essas complicações em 2010 foi 27,7\% maior em homens na faixa etária de 20 a 49 anos e 24,6\% maior em mulheres na faixa etária de 65 ou mais anos. Considerando-se todas as idades, a razão de taxas feminina/masculina demonstra que a mortalidade foi $4,9 \%$ maior no sexo feminino.

A Figura 2 apresenta as taxas de mortalidade por região, antes e após as correções para causas mal definidas de óbitos e sub-registro. As regiões com menor mortalidade por complicações agudas do diabetes em 2010 foram as Centro-Oeste, Sudeste e Sul. As regiões Nordeste e Norte apresentaram mortalidade significativamente maior, especialmente após as correções efetuadas. Nos menores de 40 anos de idade, a mortalidade por essas complicações foi significativamente menor na região Sul, em comparação com as demais regiões do país (Tabela 2 ).

\section{Tabela 1 - Óbitos declarados por complicações agudas do diabetes melito segundo código da CID-10ª e faixa etária, Brasil, 2010}

\begin{tabular}{|c|c|c|c|c|c|}
\hline Código & Código & $\begin{array}{l}<40 \text { anos } \\
\mathrm{N}(\%)\end{array}$ & $\begin{array}{l}\text { 40-59 anos } \\
\mathrm{N}(\%)\end{array}$ & $\begin{array}{l}\geq 60 \text { anos } \\
N(\%)\end{array}$ & Total \\
\hline E10.0 & Diabetes melito insulinodependente com coma ${ }^{b}$ & $17(5,4)$ & $30(3,7)$ & $117(4,5)$ & $164(4,4)$ \\
\hline E10.1 & Diabetes melito insulinodependente com cetoacidose ${ }^{c}$ & $47(15,0)$ & $62(7,6)$ & $108(4,1)$ & $217(5,8)$ \\
\hline E11.0 & Diabetes melito não insulinodependente com coma ${ }^{b}$ & $5(1,6)$ & $26(3,2)$ & $158(6,0)$ & $189(5,1)$ \\
\hline E11.1 & Diabetes melito não insulinodependente com cetoacidose ${ }^{c}$ & $4(1,3)$ & $20(2,5)$ & $112(4,3)$ & $136(3,6)$ \\
\hline $\begin{array}{l}\text { E12.0 e } \\
\text { E13.0 }\end{array}$ & $\begin{array}{l}\text { Diabetes melito relacionado com a desnutrição e outros tipos } \\
\text { especificados de diabetes melito com coma }{ }^{b}\end{array}$ & - & $4(0,5)$ & $22(0,8)$ & $26(0,7)$ \\
\hline $\begin{array}{l}\text { E12.1 e } \\
\text { E13.1 }\end{array}$ & $\begin{array}{l}\text { Diabetes melito relacionado com a desnutrição e outros tipos } \\
\text { especificados de diabetes melito com cetoacidose }{ }^{c}\end{array}$ & $3(0,9)$ & $4(0,5)$ & $13(0,5)$ & $20(0,6)$ \\
\hline E14.0 & Diabetes melito não especificado com coma ${ }^{b}$ & $60(19,2)$ & $283(34,9)$ & $1.113(42,5)$ & $1.456(38,9)$ \\
\hline E14.1 & Diabetes melito não especificado com cetoacidose ${ }^{c}$ & $177(56,5)$ & $383(47,2)$ & $973(37,2)$ & $1.533(41,0)$ \\
\hline Total & & $313(100,0)$ & $812(100,0)$ & $2.616(100,0)$ & $3.741(100,0)$ \\
\hline
\end{tabular}


As menores médias de taxas de mortalidade anuais no período de 2006 a 2010 ocorreram, em ordem crescente, nas unidades federativas do Distrito Federal, Rio Grande do Sul, São Paulo, Amapá e Mato Grosso; e as maiores médias, nos estados de Alagoas, Acre, Maranhão, Pará e Paraíba (Tabela 3). Piauí e Rio de
Janeiro apresentaram as maiores taxas de mortalidade em menores de 40 anos de idade. A taxa de mortalidade média anual variou entre as unidades federativas, de 1,02 a 5,72/100 mil habitantes; considerando-se apenas os óbitos com idade inferior a 40 anos, essa taxa variou de 0,14 a $0,60 / 100$ mil habitantes.

\section{Tabela 2 - Mortalidade por complicações agudas do diabetes melito segundo sexo e região, Brasil, 2010 e 2006-2010ª}

\begin{tabular}{|c|c|c|c|c|c|c|}
\hline \multirow{2}{*}{ Região } & \multicolumn{2}{|c|}{ Homens } & \multicolumn{2}{|c|}{ Mulheres } & \multicolumn{2}{|r|}{ Total } \\
\hline & $\mathrm{N}^{\mathrm{b}}$ & Taxa $^{c}$ & $\mathrm{~N}^{\mathrm{b}}$ & Taxa $^{c}$ & $N^{b}$ & Taxa $^{c}$ \\
\hline \multicolumn{7}{|l|}{ Geral } \\
\hline Centro-0este & 85 & $1,72(1,41-2,03)$ & 102 & $2,04(1,70-2,37)$ & 187 & $1,88(1,65-2,11)$ \\
\hline Sudeste & 608 & $1,89(1,75-2,03)$ & 797 & $1,89(1,76-2,03)$ & 1.405 & $1,89(1,80-1,99)$ \\
\hline Sul & 222 & $2,01(1,77-2,24)$ & 289 & $2,00(1,76-2,23)$ & 511 & $2,00(1,83-2,17)$ \\
\hline Nordeste & 546 & $3,23(3,01-3,45)$ & 791 & $3,68(3,45-3,91)$ & 1.337 & $3,46(3,30-3,62)$ \\
\hline Norte & 139 & $4,17(3,72-4,61)$ & 162 & $4,49(4,02-4,96)$ & 301 & $4,33(4,00-4,65)$ \\
\hline Brasil & 1.600 & $2,39(2,29-2,49)$ & 2.141 & $2,51(2,41-2,61)$ & 3.741 & $2,45(2,38-2,52)$ \\
\hline \multicolumn{7}{|c|}{$<40$ anos de idade ${ }^{c}$} \\
\hline Sul & 15 & $0,20(0,16-0,24)$ & 15 & $0,22(0,18-0,27)$ & 30 & $0,21(0,18-0,24)$ \\
\hline Centro-0este & 15 & $0,35(0,28-0,43)$ & 9 & $0,25(0,19-0,31)$ & 24 & $0,30(0,25-0,35)$ \\
\hline Sudeste & 67 & $0,30(0,23-0,37)$ & 76 & $0,31(0,25-0,38)$ & 143 & $0,31(0,26-0,36)$ \\
\hline Norte & 14 & $0,39(0,32-0,46)$ & 7 & $0,24(0,18-0,30)$ & 21 & $0,32(0,27-0,36)$ \\
\hline Nordeste & 46 & $0,35(0,26-0,43)$ & 49 & $0,33(0,25-0,41)$ & 95 & $0,34(0,28-0,40)$ \\
\hline Brasil & 157 & $0,31(0,27-0,35)$ & 156 & $0,28(0,23-0,32)$ & 313 & $0,29(0,26-0,32)$ \\
\hline
\end{tabular}

a) Em menores de 40 anos de idade, como houve - relativamente - poucos óbitos em 2010 nas regiōes Sul, Centro-Oeste e Norte, foi utilizada a média das taxas anuais de 2006 a 2010 para as regiões b) Número de óbitos em 2010

c) Taxa de mortalidade corrigida por óbitos de causa mal definida e não declarados, padronizada por idade, por 100 mil habitantes/ano (intervalo de confiança de 95\%).

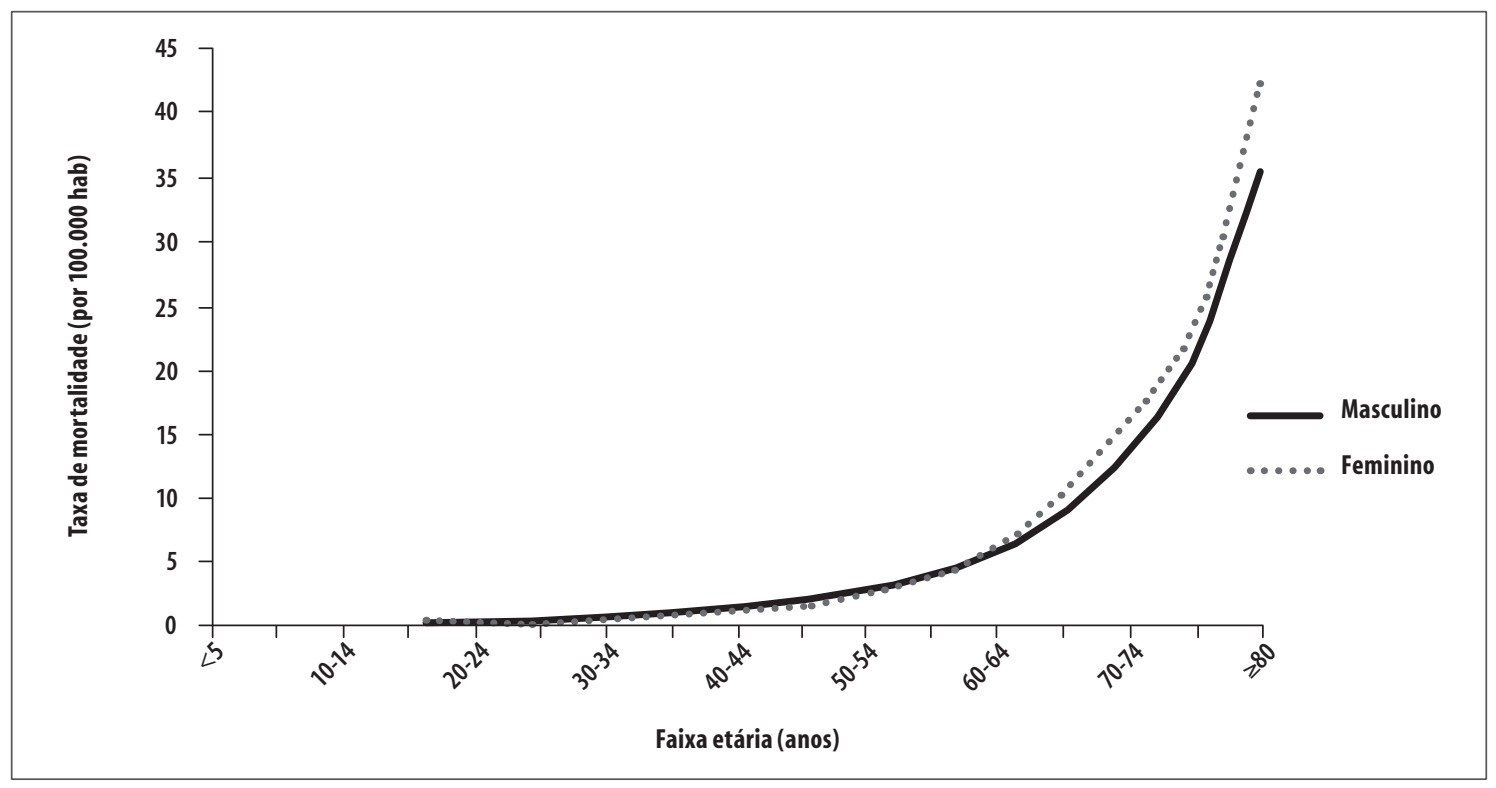

Figura 1 - Mortalidade por complicações agudas do diabetes melito segundo faixa etária e sexo, padronizada por idade e corrigida para a presença de causas mal definidas de óbitos e sub-registro, Brasil, 2010 


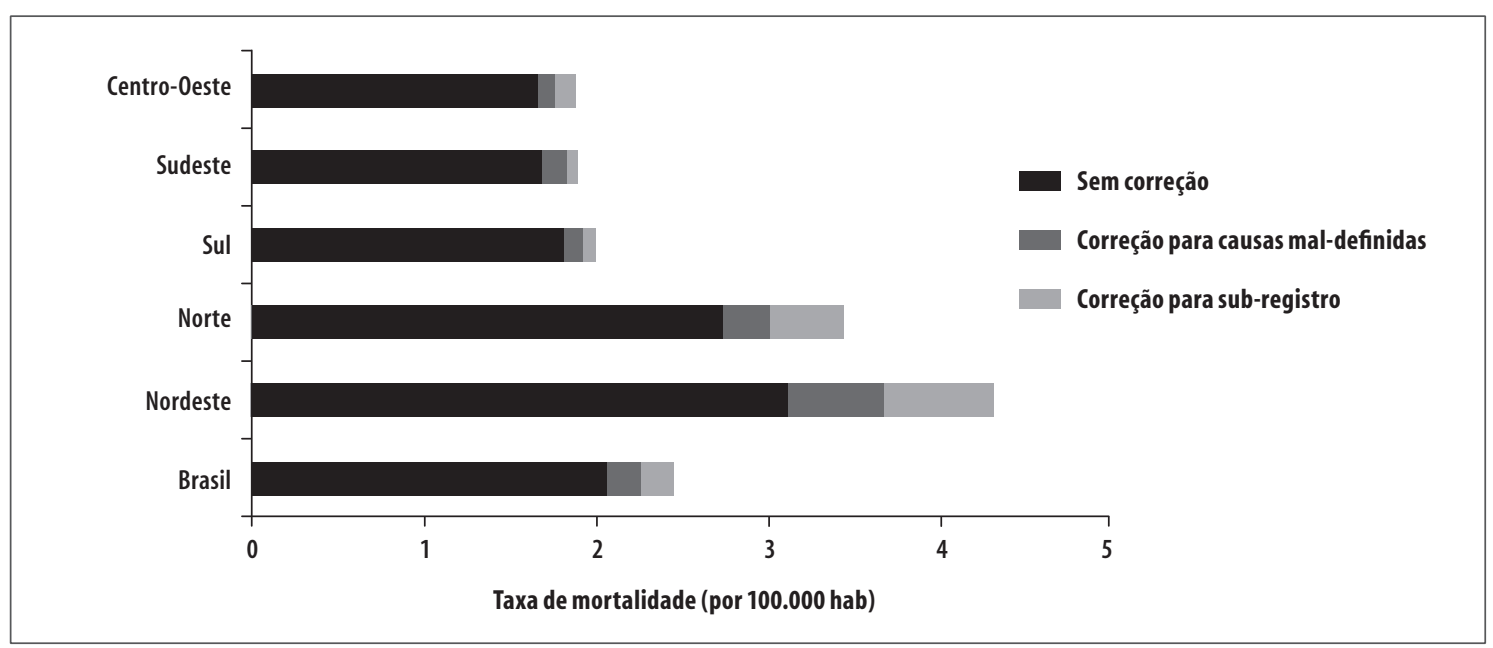

Figura 2 - Mortalidade por complicações agudas do diabetes melito segundo região, padronizada por idade, com e sem correção pelos óbitos por causas mal definidas e óbitos não declarados, Brasil, 2010

Tabela 3 - Mortalidade por complicações agudas do diabetes melito na população geral e em menores de 40 anos de idade, segundo unidade da federação (UF), Brasil, 2006-2010 ${ }^{\mathrm{b}}$

\begin{tabular}{|c|c|c|c|c|}
\hline \multirow{2}{*}{ UF } & \multicolumn{2}{|c|}{ Geral } & \multicolumn{2}{|c|}{$<40$ anos } \\
\hline & $\mathrm{N}^{\mathrm{a}}$ & $\operatorname{Taxa}^{b}$ & $\mathrm{~N}^{\mathrm{a}}$ & $\operatorname{Taxa}^{b}$ \\
\hline Distrito Federal & 21 & $1,02(0,84-1,20)$ & 7 & $0,18(0,09-0,27)$ \\
\hline Rio Grande do Sul & 135 & $1,23(1,13-1,32)$ & 13 & $0,15(0,11-0,20)$ \\
\hline São Paulo & 554 & $1,50(1,45-1,55)$ & 52 & $0,17(0,15-0,20)$ \\
\hline Amapá & 8 & $1,91(1,43-2,39)$ & 1 & $0,19(0,02-0,36)$ \\
\hline Mato Grosso & 42 & $2,09(1,86-2,33)$ & 3 & $0,27(0,17-0,36)$ \\
\hline Santa Catarina & 157 & $2,15(1,98-2,31)$ & 6 & $0,14(0,09-0,19)$ \\
\hline Minas Gerais & 351 & $2,19(2,10-2,28)$ & 37 & $0,41(0,36-0,46)$ \\
\hline Espírito Santo & 63 & $2,24(2,02-2,46)$ & 6 & $0,27(0,17-0,36)$ \\
\hline Mato Grosso do Sul & 32 & $2,35(2,08-2,63)$ & 4 & $0,38(0,25-0,52)$ \\
\hline Paraná & 219 & $2,37(2,24-2,51)$ & 11 & $0,31(0,25-0,37)$ \\
\hline Goiás & 92 & $2,38(2,20-2,56)$ & 10 & $0,34(0,26-0,42)$ \\
\hline Ceará & 165 & $2,58(2,43-2,74)$ & 7 & $0,26(0,21-0,32)$ \\
\hline Rio Grande do Norte & 66 & $2,67(2,42-2,93)$ & 5 & $0,27(0,18-0,37)$ \\
\hline Amazonas & 33 & $2,75(2,49-3,00)$ & 1 & $0,25(0,16-0,33)$ \\
\hline Rio de Janeiro & 437 & $2,94(2,82-3,06)$ & 48 & $0,57(0,51-0,64)$ \\
\hline Pernambuco & 179 & $2,94(2,78-3,10)$ & 14 & $0,30(0,24-0,37)$ \\
\hline Rondônia & 26 & $3,13(2,74-3,53)$ & 3 & $0,32(0,17-0,46)$ \\
\hline Bahia & 311 & $3,37(3,24-3,51)$ & 27 & $0,42(0,36-0,48)$ \\
\hline Roraima & 5 & $3,38(2,60-4,16)$ & 0 & $0,24(0,00-0,47)$ \\
\hline Sergipe & 38 & $3,44(3,07-3,80)$ & 9 & $0,41(0,26-0,55)$ \\
\hline Piauí & 102 & $4,25(3,92-4,58)$ & 10 & $0,60(0,45-0,74)$ \\
\hline Tocantins & 33 & $4,44(3,93-4,94)$ & 1 & $0,34(0,17-0,50)$ \\
\hline Alagoas & 107 & $4,47(4,14-4,81)$ & 6 & $0,35(0,24-0,46)$ \\
\hline Acre & 19 & $4,53(3,82-5,23)$ & 1 & $0,18(0,02-0,34)$ \\
\hline Maranhão & 173 & $4,94(4,70-5,19)$ & 13 & $0,41(0,33-0,49)$ \\
\hline Pará & 177 & $5,36(5,13-5,60)$ & 14 & $0,37(0,30-0,45)$ \\
\hline Paraíba & 196 & $5,72(5,38-6,07)$ & 4 & $0,39(0,28-0,50)$ \\
\hline
\end{tabular}

a) Total de óbitos declarados em 2010

b) Média da taxa de mortalidade anual no período de 2006 a 2010, padronizada por idade e corrigida pelos óbitos por causas mal definidas e năo declarados, por 100 mil habitantes (intervalo de confiança de 95\%). 


\section{Discussão}

A mortalidade por complicações agudas do diabetes no Brasil em 2010 foi de 2,45 óbitos por 100 mil habitantes, representando 6,8\% do total dos óbitos com menção de diabetes. Considerando-se apenas os menores de 40 anos, a taxa de mortalidade foi de 0,29 por 100 mil habitantes, representando $22,9 \%$ dos óbitos com menção de diabetes nessa faixa etária. 0 grande potencial de evitabilidade dessas complicações indica que esses coeficientes são elevados, especialmente nas regiões Nordeste e Norte, sugerindo a necessidade de intensificação de ações preventivas e maior acesso ao diagnóstico e pronto tratamento dessas complicações.

A taxa de mortalidade por complicações agudas do diabetes aumentou exponencialmente com a idade, o que está de acordo com os dados da literatura. ${ }^{5,6,12-14} A$ associação decorre da maior mortalidade geral nas faixas etárias mais idosas, quando múltiplas causas de adoecimento e morte podem se apresentar. Entre as causas de complicações agudas fatais do diabetes estão as infecções respiratórias, 0 infarto do miocárdio e 0 acidente vascular cerebral. Para os jovens, aspectos da organização dos serviços de saúde como a adequada distribuição de insulina, o pronto atendimento às descompensações agudas e o cuidado continuado e integral das pessoas com diabetes podem reduzir a mortalidade devida a essas causas até valores próximos de zero, constituindo-se, portanto, em um bom indicador da qualidade dos serviços de saúde. Os dados deste estudo indicaram uma variação de $0,14 / 100$ mil hab. (Santa Catarina) a 0,60/100 mil hab. (Piauí) nesse indicador entre os mais jovens, ilustrando a magnitude das iniquidades geográficas.

Poucos estudos publicados apresentam avaliação da taxa de mortalidade por complicações agudas do diabetes na população. ${ }^{6,12,14-16}$ Para o Brasil, não foram encontrados artigos com dados populacionais sobre 0 assunto. A taxa de mortalidade encontrada para 0 país foi, aproximadamente, três vezes maior que a dos Estados Unidos da América (EUA) em 2009 (0,75 por 100 mil hab.) $;{ }^{15}$ e também maior que a mortalidade por acidose ou coma nos mesmos EUA em 1970 (1,4 por 100 mil hab.). ${ }^{12}$ Considerando-se apenas os mais jovens, para os quais a mortalidade é quase inteiramente evitável, a comparação dos dados do presente estudo com os obtidos nos EUA também indicam que a mortalidade por diabetes no Brasil pode ser reduzida. No estado de Washington/EUA, a mortalidade por complicações agudas do diabetes em menores de 45 anos de idade era de 0,39 óbitos por 100 mil habitantes entre 1968 e 1979, apenas um pouco superior à verificada em 2010 no Brasil (0,29/100 mil hab.), em faixa etária semelhante (menores de 40 anos) ${ }^{16}$

As regiões Nordeste e Norte apresentaram maior mortalidade, independentemente da faixa etária (à exceção, talvez, dos estados do Ceará, Rio Grande do Norte e Amazonas). Esses resultados gerais vão ao encontro dos principais indicadores demográficos, socioeconômicos, de recursos e de cobertura dos serviços de Saúde Pública nas regiões brasileiras em 2010, como, por exemplo, os indicadores apresentados nos 'Indicadores e Dados Básicos do Brasil de 2011' (IDB 2011) ${ }^{17}$ e sugerem que as desigualdades nesses indicadores traduzem-se em (i) menor conhecimento sobre diabetes e seu manejo e (ii) pior qualidade de manejo clínico (diagnóstico, tratamento continuado e atendimento das crises agudas).

As diferenças observadas entre homens e mulheres merecem atenção. A maior taxa de mortalidade feminina pode ser atribuída, principalmente, à maior mortalidade neste sexo após os 65 anos de idade, o que também foi observado em outros estudos. Nos EUA, a mortalidade por acidose ou coma no período de 1970 a 1978 foi maior em mulheres, de 1,4 óbito por 100 mil habitantes, frente a uma taxa de 1,1 por 100 mil hab. em homens. ${ }^{12}$ Os autores ponderam que possíveis explicações para esse achado seriam maior diagnóstico, tratamento e notificação do diabetes nas declarações de óbito no sexo feminino. Estudo realizado no estado de São Paulo em 1992, ao avaliar a mortalidade por diabetes em geral, encontrou maior mortalidade pela doença no sexo feminino, no grupo etário de 40 ou mais anos, ${ }^{18}$ inferindo também que isso possa ter ocorrido em função do maior conhecimento do diagnóstico de diabetes em mulheres e, por conseguinte, favorecido a menção do diagnóstico de diabetes nas declarações de óbitos femininos.

Maior taxa de mortalidade em mulheres nas faixas etárias mais avançadas também foi observada nos EUA entre 1970 e $1978 .{ }^{12}$ Um estudo de coorte britânico identificou diabéticos em uso de insulina antes dos 30 anos de idade, entre 1972 e 1993, e os acompanhou até 1997, encontrando maior mortalidade no sexo masculino apenas entre os 20 e os 49 anos, ${ }^{19}$ da mesma forma que os resultados do presente estudo. Por sua vez, o estudo norte-americano supracitado, ao avaliar a mortalidade por diabetes com acidose ou coma, utilizou faixas etárias bem mais amplas e observou 
maior mortalidade por essas complicações no sexo masculino apenas na faixa etária de 15 a 44 anos. ${ }^{12}$

Ao considerar a potencial evitabilidade dessa causa de mortalidade e as taxas observadas nos EUA, os dados aqui apresentados apontam que, apesar das inegáveis melhorias na saúde da população brasileira em geral ${ }^{20}$ e em relação às doenças crônicas não transmissíveis (DCNT) mais especificamente, ${ }^{21}$ ainda há um amplo espaço para ações de prevenção e de controle das complicações agudas do diabetes e para redução das iniquidades regionais e sociais de saúde. Nesse sentido, o Sistema Único de Saúde (SUS), assim como a Saúde Suplementar, está passando por uma transição organizacional: de uma Atenção Primária focada em condições agudas para uma atenção capaz de manejar condições crônicas, caso do diabetes. ${ }^{22,23}$ Encontramse em planejamento redes que integram os diferentes níveis de atenção, ${ }^{24}$ capazes de facilitar a prevenção e 0 pronto-atendimento das descompensações agudas do diabetes, além da coordenação do cuidado prestado.

Ademais, os dados deste estudo demonstram a importância da utilização de indicadores de mortalidade na avaliação dos serviços de saúde. Considerando-se a obrigatoriedade de seu registro, o que facilita sua cobertura, e a ampla padronização internacional, é surpreendente a pouca utilização desse indicador em nível global. ${ }^{25}$

0 presente estudo apresenta limitações. 0 Sistema de Informações sobre Mortalidade - SIM - brasileiro todavia apresentava, em 2010, uma subnotificação dos óbitos de $5,8 \%{ }^{26}$ e, entre óbitos declarados, significativa proporção de óbitos por causas mal definidas, da ordem de $7,0 \%{ }^{27} \mathrm{~A}$ correção realizada para óbitos declarados por causas mal definidas e para sub-registro visa a atenuar essas eventuais falhas, embora as estimativas ainda sejam imperfeitas. Melhorias em curso no SIM tendem a reduzir esse problema para os próximos anos. Igualmente digno de nota é o elevado percentual dos

\section{Referências}

1. International Diabetes Federation. IDF Diabetes Atlas. 6th ed. [Internet]. Brussels: IDF; 2013 [cited 2013 Dec 3]. Available from: http://www.idf.org/diabetesatlas

2. American Diabetes Association. Hyperglycemic crises in patients with diabetes mellitus. Diabetes Care. 2001 Nov;24(11):1988-96.

3. Kitabchi AE, Umpierrez GE, Miles JM, Fisher JN. Hyperglycemic crises in adult patients with diabetes. Diabetes Care. 2009 Jul;32(7):1335-43. óbitos sem determinação do tipo de diabetes, ilustrando a necessidade de melhorias na definição da causa básica do SIM no que tange a essa doença.

A mortalidade por complicações agudas do diabetes mostrou-se um indicador simples e sintético dos cuidados oferecidos aos pacientes com diabetes no país. Considerando-se que essas complicações são em grande parte evitáveis mediante adequada educação e atendimento, as taxas de mortalidade mais elevadas no Norte e Nordeste indicam marcantes iniquidades regionais. A taxa encontrada para o Brasil, bem maior do que aquela observada em países de alta renda, revela um amplo espaço para o aprimoramento geral dos cuidados aos pacientes com diabetes no país.

\section{Agradecimentos}

Ao apoio financeiro do Termo de Cooperação ${ }^{\circ}$ 80/2009, entre a UFRGS e o Ministério da Saúde.

\section{Contribuição dos autores}

Klafke A contribuiu na concepção e delineamento do estudo, análise e interpretação os dados, redação e revisão crítica do conteúdo intelectual do artigo.

Duncan BB contribuiu na concepção e delineamento do estudo, análise e interpretação os dados, e revisão crítica do conteúdo intelectual do artigo.

Schmidt MI contribuiu na concepção e delineamento do estudo, interpretação dos dados e revisão crítica do conteúdo intelectual do artigo.

Rosa RS, Moura L e Malta DC contribuíram na revisão crítica do conteúdo.

Todos os autores aprovaram a versão final do manuscrito e são responsáveis por todos os aspectos do trabalho, incluindo a garantia de sua precisão e integridade.

4. Gaglia JL, Wyckoff J, Abrahamson MJ. Acute hyperglycemic crisis in the elderly. Med Clin North Am. 2004 Jul;88(4):1063-84.

5. Kitabchi AE, Umpierrez GE, Murphy MB, Barrett EJ, Kreisberg RA, Malone JI, et al. Management of hyperglycemic crises in patients with diabetes. Diabetes Care. 2001 Jan;24(1):131-53.

6. Snorgaard O, Eskildsen PC, Vadstrup S, Nerup J. Diabetic ketoacidosis in Denmark: epidemiology, 
incidence rates, precipitating factors and mortality rates. J Intern Med. 1989 Oct;226(4):223-8.

7. United Nations. Political declaration of the High-level Meeting of the General Assembly on the Prevention and Control of Non-communicable Diseases. Washington, DC: United Nations; 2011.

8. Mathers CD, Bernard C, Iburg KM, Inoue M, Fat DM, Shibuya K, et al. Global Burden of Disease in 2002: data sources, methods and results. Geneva: World Health Organization; 2003.

9. Victora CG, Aquino EML, Leal MC, Monteiro CA, Barros FC, Szwarcwald CL. Maternal and child health in Brazil: progress and challenges. Lancet. 2011 May;377(9780):1863-76.

10. Stevens AP. Tendências na mortalidade por doenças crônicas não-transmissíveis no Brasil: métodos computacionais aplicados aos anos de 1991 a 2010 [tese]. Porto Alegre (RS): Universidade Federal do Rio Grande do Sul; 2012.

11. Dever GEA. Medidas epidemiológicas. In: Dever GEA, Champagne F, editores. A epidemiologia na administração dos serviços de saúde. São Paulo: Pioneira; 1988. p. 71-107.

12. Holman RC, Herron CA, Sinnock P. Epidemiologic characteristics of mortality from diabetes with acidosis or coma, United States, 1970-78. Am J Public Health. 1983 Oct;73(10):1169-73.

13. MacIsaac RJ, Lee LY, McNeil KJ, Tsalamandris C, Jerums G. Influence of age on the presentation and outcome of acidotic and hyperosmolar diabetic emergencies. Intern Med J. 2002 Aug;32(8):379-85.

14. Henriksen OM, Roder ME, Prahl JB, Svendsen OL. Diabetic ketoacidosis in Denmark: incidence and mortality estimated from public health registries. Diabetes Res Clin Pract. 2007 Apr;76(1):51-6.

15. Centers for Disease Control and Prevention. National Center for Chronic Disease Prevention and Health Promotion. Division of Diabetes Translation. Mortality due to hyperglycemic crises [Internet]. Atlanta (GA): Centers for Disease Control and Prevention; 2012 Nov [cited 2013 Jul 18]. Available from: http://www.cdc. gov/diabetes/statistics/complications_national.htm\#2

16. Connell FA, Louden JM. Diabetes mortality in persons under 45 years of age. Am J Public Health. 1983 Oct;73(10):1174-77.

17. Ministério da Saúde (BR). Rede Interagencial de Informação para a Saúde. Indicadores e dados básicos: Brasil - 2011 [Internet]. Brasília: RIPSA; 2012 [citado 2013 jul 18]. Disponível em: http:// tabnet.datasus.gov.br/cgi/idb2011/matriz.htm
18. Franco LJ, Mameri C, Pagliaro H, Iochida LC, Goldenberg P. Diabetes as primary or associated cause of death in the state of São Paulo, Brazil, 1992. Rev Saude Publica. 1998 Jun;32(3):237-45.

19. Laing SP, Swerdlow AJ, Slater SD, Botha JL, Burden AC, Waugh NR, et al. The British Diabetic Association Cohort Study, II: cause-specific mortality in patients with insulin-treated diabetes mellitus. Diabet Med. 1999 Jun;16(6):466-71.

20. Victora CG, Barreto ML, Leal MC, Monteiro CA, Schmidt MI, Paim J, et al. Health conditions and health-policy innovations in Brazil: the way forward. Lancet. 2011 Jun;377(9782):2042-53.

21. Schmidt MI, Duncan BB, Silva GA, Menezes AM, Monteiro CA, Barreto SM, et al. Chronic noncommunicable diseases in Brazil: burden and current challenges. Lancet. 2011 Jun;377(9781):1949-61.

22. Duncan MS, Goldraich MA, Chueiri PS. Cuidados longitudinais e integrais a pessoas com condições crônicas. In: Duncan BB, Schmidt MI, Giugliani EJ, Duncan MS, Giugliani C, editores. Medicina ambulatorial: condutas de atenção primária baseadas em evidências. 4. ed. Porto Alegre: Artmed; 2013. p. 892-904.

23. Mendes EV. O cuidado das condições crônicas na atenção primária à saúde: 0 imperativo da consolidação da estratégia da saúde da família [Internet]. Brasília: Organização Pan-Americana da Saúde; 2012 [citado 2013 ago 14]. Disponível em: http://apsredes.org/ site2012/wp-content/uploads/2012/04/Redes-deAtencao-condicoes-cronicas.pdf

24. Brasil. Ministério da Saúde. Portaria n ${ }^{0} 4.279$, de 30 de dezembro de 2010. Estabelece diretrizes para a organização da Rede de Atenção à Saúde no âmbito do Sistema Único de Saúde (SUS). Diário Oficial da República Federativa do Brasil, Brasília (DF), 2010 dez 31; Seção 1:89.

25. Vermelho LL, Costa AJ, Kale PL. Indicadores de saúde. In: Medronho RA, Carvalho DM, Bloch KV, Luiz RR, Werneck GL, editores. Epidemiologia. São Paulo: Atheneu; 2004. p. 33-55.

26. Ministério da Saúde (BR). Rede Interagencial de Informação para a Saúde. Indicadores de Cobertura. Razão entre óbitos informados e estimados [Internet]. Brasília: RIPSA; 2011 [citado 2013 ago 5]. Disponível em: http://tabnet.datasus.gov.br/cgi/idb2011/a18.htm

27. Ministério da Saúde (BR). Secretaria de Vigilância em Saúde. Departamento de Análise da Situação de Saúde. Mortalidade - Brasil [Internet]. Brasília: DATASUS; 2012 [citado 2013 ago 5]. Disponível em: http://tabnet. datasus.gov.br/cgi/deftohtm.exe?sim/cnv/obt10uf.def

Recebido em 19/01/2014

Aprovado em 07/06/2014 